TITLE:

\title{
Radiation-induced graft polymerization of amphiphilic monomers with different polymerization characteristics onto hydrophobic polysilane
}

\section{$\operatorname{AUTHOR}(S):$}

Tanaka, Hidenori; Iwasaki, Isao; Kunai, Yuichiro; Sato, Nobuhiro; Matsuyama, Tomochika

\section{CITATION:}

Tanaka, Hidenori ... [et al]. Radiation-induced graft polymerization of amphiphilic monomers with different polymerization characteristics onto hydrophobic polysilane. Radiation Physics and Chemistry 2011, 80(8): 884-889

\section{ISSUE DATE:}

2011-08

URL:

http://hdl.handle.net/2433/141840

\section{RIGHT:}

C 2011 Elsevier Ltd.; この論文は出版社版でありません。引用の際には 出版社版をご確認ご利用ください。; This is not the published version. Please cite only the published version. 


\section{Radiation-induced Graft Polymerization of Amphiphilic Monomers with Different Polymerization Characteristics onto Hydrophobic Polysilane}

Hidenori Tanaka, Isao Iwasaki, Yuichiro Kunai, Nobuhiro Sato*, and Tomochika Matsuyama

Research Reactor Institute, Kyoto University, Asashironishi 2-1010, Kumatori-cho, Sennan-gun, Osaka 590-0494, Japan

Corresponding author : Nobuhiro Sato

e-mail : sato-n@rri.kyoto-u.ac.jp

Phone: +81-72-451-2499

Fax: +81-72-451-2633

\section{Abstract}

The structures of poly(methyl- $n$-propylsilane) (PMPrS) amphiphilically modified through $\gamma$-ray-induced graft polymerization were investigated with ${ }^{1} \mathrm{H}$ NMR measurement. By use of methyl methacrylate (MMA) or diethyl fumarate (DEF) as 
monomers for the graft polymerization, grafting yield rose with increasing total absorption dose and monomer concentrations, but decreased with increasing dose rate. This result means that grafting yield of modified PMPrS can be controlled by changing irradiation conditions. However, the number of PMMA or PDEF graft chains per PMPrS chain was estimated to be less than 1.0 by analysis of ${ }^{1} \mathrm{H}$ NMR spectra, and this value was lower than we had expected. To improve graft density, maleic anhydride (MAH), which is known as a non-homopolymerizable monomer in radical polymerization, was used as a monomer for grafting. As a result, high density grafting (one MAH unit for 4.2 silicon atoms) was attained. It demonstrates that the structure of $\gamma$-ray-modified polysilane strongly depends on the polymerization characteristics of grafted monomers.

\section{Keywords}

Polysilanes / Graft Copolymers / $\gamma$-Rays / Radiation Modification

\section{Introduction}

Polysilane has attracted much attention because it has unique properties arising from delocalized electrons along the silicon backbone (Miller and Michl, 1989; West, 1986, 
1992). If polysilane is applied to fabricate ordered materials in the nanometer scale, further unique properties can be developed. The utilization of self-assemblies is considered as powerful methods for preparing ordered structures. Since it is widely known that amphiphilic block or graft copolymers can form self-assemblies such as micelles and oriented Langmuir monolayers (Alexandridis and Lindman, 2000; Riess, 2003; Webber et al., 1996), amphiphilic polysilane copolymers are also expected to serve as ordered materials.

For the synthesis of polysilane copolymers with hydrophilic or amphiphilic chains, we can consider the following two approaches: the modification of presynthesized hydrophobic polysilane and the direct polymerization of monomeric silanes with hydrophilic or amphiphilic groups. In the latter case, however, the Wurtz-type coupling reaction, which is commonly used for the synthesis of polysilane, can not be applied because sodium metal used in the reaction violently attacks reactive functional groups (Miller and Michl, 1989; West, 1986). Thus, this way is limited in its use. In contrast, the former way is free from such a limitation. Therefore we studied the modification of presynthesized polysilane to obtain polysilane-based amphiphilic graft copolymers by utilizing $\gamma$-ray-induced graft polymerization.

In the present study, we investigated the structures of amphiphilic graft polysilane 
modified under various irradiation conditions to establish optimized irradiation. As a hydrophobic base polymer for the graft polymerization, poly(methyl-n-propylsilane) (PMPrS) was employed. For grafted molecules, three types of monomers bearing hydrophilic groups were used: methyl methacrylate (MMA), diethyl fumarate (DEF) and maleic anhydride (MAH). MMA and DEF are homopolymerizable monomers having different propagation rate constants, while MAH is known as a non-homopolymerizable monomer in radical polymerization (Hodge et al., 1985, 1990). It can be expected that the structure and property of modified PMPrS differ according to the polymerization characteristics of these grafted monomers. In each experiment performed with the grafting monomers, we tried to control grafting yield, which is defined as monomer units of grafted chains per silicon atom of PMPrS, by changing irradiation conditions such as total absorption dose, dose rate and the concentrations of grafting monomers.

\section{Experimental}

\subsection{Monomers.}

Methyl-n-propyldichlorosilane (Shin-Etsu Chemical, Co.), methyl methacrylate (MMA) (Nakalai Tesque, Inc.) and diethyl fumarate (DEF) (Nakalai Tesque, Inc.) were 
used after purification by reduced-pressure distillation. Maleic anhydride (MAH) was employed after purification by re-crystallization with toluene.

\subsection{Synthesis of PMPrS.}

PMPrS was synthesized via the Wurtz-type coupling reaction of methyl- $n$-propyldichlorosilane. Crude PMPrS was purified by re-precipitation from a toluene solution into methanol, and then pure PMPrS was obtained by freeze-drying from a benzene solution. The number average molecular weight of PMPrS used in the following experiments is listed in Table 1. PMPrS1, PMPrS2 and PMPrS3 were employed for grafting poly(methyl methacrylate) (PMMA), poly(diethyl fumarate) (PDEF) and MAH, respectively.

\section{Table 1.}

\subsection{Radiation-modification of PMPrS.}

PMMA-grafted PMPrS (PMPrS-g-PMMA), PDEF-grafted PMPrS (PMPrS-g-PDEF) and MAH-grafted PMPrS (PMPrS- $g$-MAH) were synthesized as follows: PMPrS and each monomer were co-dissolved in toluene. The toluene solution was degassed and sealed in a glass tube, and then irradiated with ${ }^{60} \mathrm{Co}-\gamma$-rays at room temperature. 
In the case of PMPrS-g-PMMA, the toluene solution after $\gamma$-irradiation was poured into methanol to precipitate a product. To remove PMMA homopolymer contained in the crude product, the precipitate was purified by fractional precipitation with acetone/methanol. Acetone is a good solvent for PMMA but a poor solvent for PMPrS, and methanol is a poor solvent for both PMMA and PMPrS. When a small amount of a toluene solution of the crude product was dropped into a large amount of acetone, PMPrS-g-PMMA formed emulsions. By adding methanol into the acetone solution containing the emulsions of the product, four regimes were found according to the methanol amount. In the first regime, no changes were recognized in spite of methanol addition. In the second regime, however, further methanol addition caused the solution more opaque and the amount of centrifuged precipitates was increased. In the third regime, the amount of precipitates was constant independently of the methanol amount, but in the fourth regime, precipitates obtained were again increased with increasing methanol amount. PMPrS-g-PMMA was successfully recovered in the second regime. After the fractional precipitation, PMPrS- $g$-PMMA was freeze-dried with benzene.

The purity of PMPrS-g-PMMA obtained was verified by iodine-staining thin-layer chromatography (TLC) with a silica plate and an eluent of a tetrahydrofuran (THF)/toluene $(4 / 6, \mathrm{v} / \mathrm{v})$ mixture. The eluent could separate PMPrS-g-PMMA and 
PMMA homopolymer completely. In the TLC experiment with purified PMPrS-g-PMMA, we could not recognize the spot of PMMA homopolymer. This fact indicates that the amount of PMMA contained was under the detection limit in the purified product. The detection limit of PMMA was determined in another TLC experiment, and finally the content of PMMA homopolymer in PMPrS-g-PMMA was found to be less than $1.0 \mathrm{wt} \%$.

In the case of PMPrS-g-PDEF, solvent toluene was removed by evaporation from the reaction solution after irradiation, and then crude PMPrS- $g$-PDEF was freeze-dried from a benzene solution. Next, PMPrS-g-PDEF was precipitated from a THF solution into water, and recovered again by freeze drying after unreacted DEF monomers contained in the precipitate were removed by filtration. This reprecipitation procedure was repeated 3 - 5 times. Some products containing PDEF homopolymer above $1.0 \mathrm{wt} \%$ were further purified by fractional precipitation with THF/hexane. THF is a good solvent for both PMPrS and PDEF, while hexane is a good solvent only for PMPrS but not for PDEF. When hexane is added to the THF solution of the product, PMPrS-g-PDEF forms emulsions and PDEF homopolymer precipitates. Accordingly, PDEF homopolymer could be removed by centrifugation after successive addition of hexane. Finally, the product was freeze-dried twice from a benzene solution and then 
vacuum-dried for 40 hours to remove water completely.

The purity of PMPrS-g-PDEF was verified by TLC with a methanol eluent and a sulfuric acid stain in the same manner as described above. The content of PDEF homopolymer was also found to be less than $1.0 \mathrm{wt} \%$.

In the case of PMPrS-g-MAH, solvent toluene was removed by evaporation from the reaction solution after irradiation. Pure PMPrS- $g$-MAH was isolated from the crude product by solvent extraction with hexane and then obtained by freeze-drying from a benzene solution. The content of unreacted MAH was checked to be less than $0.5 \mathrm{wt} \%$ by GPC with a UV detector.

\subsection{Spectroscopy.}

${ }^{1} \mathrm{H}$ NMR spectra were recorded on a JEOL EX 400 spectrometer at 25 or $55^{\circ} \mathrm{C}$ in deuterated chloroform solutions.

\subsection{Evaluation of Grafting Yield.}

Grafting yield, which we define as the number of monomer unit per silicone atom of PMPrS, was calculated from the intensity ratio of ${ }^{1} \mathrm{H}$ NMR signals. In reference to the integrated signal intensity for a methyl proton of PMPrS ( $\mathrm{SiCH}_{3}, \delta 0.25 \mathrm{ppm}$ ), the intensity rations of a methyl proton of PMMA $\left(\mathrm{COOCH}_{3}, \delta 3.7 \mathrm{ppm}\right)$ for PMPrS-g-PMMA, a methylene proton of PDEF $\left(\mathrm{COOCH}_{2} \mathrm{CH}_{3}, \delta 4.2 \mathrm{ppm}\right)$ for 
PMPrS- $g$-PDEF and a methine proton of MAH $(\mathrm{CHCH}, \delta 2.0-5.0 \mathrm{ppm})$ for PMPrS-g-MAH were calculated to evaluate their grafting yields.

\section{Results and Discussion}

\subsection{Irradiation Effect on PMPrS.}

The molecular weight of PMPrS modified with grafted chains should become larger than that of PMPrS irradiated without monomers. Therefore, we examined the change of molecular weight of PMPrS when PMPrS was irradiated by $\gamma$-rays with and without MMA monomers in toluene. Figure 1 shows the variation of molecular weight depending on dose. When PMPrS alone is irradiated with $\gamma$-rays, its molecular weight decreases with an increase of dose. This result indicates that PMPrS is degraded by $\gamma$-rays. When PMPrS is irradiated together with MMA, on the other hand, the decrease of the molecular weight is smaller. One reason is that MMA monomers shows protection effect on PMPrS degradation by absorbing $\gamma$-rays and consuming radicals. Moreover, PMMA grafting on PMPrS is also responsible for this smaller molecular weight decrease. The result obtained here suggests that $\gamma$-irradiation successfully cause the graft polymerization of MMA onto PMPrS in spite of degradation of PMPrS. 
Figure 1. Variation of molecular weight of PMPrS depending on dose. Dose rate was $3.1 \mathrm{kGy} / \mathrm{h}$. Open and closed circles indicate the molecular weight of PMPrS irradiated with and without MMA, respectively. The molecular weight was measured by GPC with RI detector.

\subsection{Grafting Yield of PMPrS-g-PMMA}

We tried to control grafting yield by changing irradiation conditions. Figure 2a shows the dependence of grafting yield of PMPrS-g-PMMA on dose at a constant dose rate of $3.1 \mathrm{kGy} / \mathrm{h}$. Although grafting yield obviously rises with an increase of dose, the increase rate of grafting yield gradually reduces at higher doses. The reason for this rate reduction is partly attributed to the consumption of MMA monomers by graftand homo-polymerization of MMA. The concentration of MMA monomers available for graft polymerization decreases at higher doses.

Figure $2 \mathrm{~b}$ exhibits that grafting yield decreases with increasing dose rate despite the total absorption dose is the same. This decrease of grafting yield is explained by PMMA homopolymerization. At higher dose rates, $\gamma$-rays generate more MMA radicals simultaneously in the reaction solution, which leads to more facile homopolymerization of MMA. Thus, MMA monomers available for graft 
polymerization diminish, and consequently grafting yield decreases in spite of the same dose.

Figure 2. Dependence of grafting yield of PMPrS-g-PMMA on (a) dose and (b) dose rate. The concentrations of PMPrS and MMA were both $10 \mathrm{wt} \%$.

Figure 3 depicts the dependence of grafting yield of PMPrS-g-PMMA on the concentration of MMA. Grafting yield rises in proportion to the MMA concentration under $40 \mathrm{wt} \%$. Above MMA concentrations of $60 \mathrm{wt} \%$, gelation took place upon irradiation and therefore it was impossible to isolate PMPrS- $g$-PMMA from the product. This indicates that crosslinking of PMMA occurs at higher concentrations.

Figure 3. Dependence of grafting yield of PMPrS-g-PMMA on the concentration of MMA. The concentration of PMPrS was $10 \mathrm{wt} \%$. Dose rate was $1.9 \mathrm{kGy} / \mathrm{h}$ and dose was 40 kGy.

Thus, we demonstrated that grafting yield of PMPrS-g-PMMA could be controlled by the irradiation conditions such as dose, dose rate and the concentration of MMA. 


\subsection{Characterization of PMPrS-g-PMMA}

Polysilane has a strong absorption band in UV region due to the $\sigma-\sigma^{*}$ transition of delocalized electrons along its backbone. However, polysilane is degraded by $\gamma$-irradiation as described above. Therefore, we checked whether or not PMPrS- $g$-PMMA keeps this electronic property after $\gamma$-irradiation from UV absorption spectra of PMPrS and PMPrS-g-PMMA. The shape of the spectra and the molar absorption coefficients were little changed by irradiation. These results indicate that the electronic property derived from the delocalized electrons along the silicon backbone is scarcely affected by the radiation-modification.

ESR and ${ }^{1} \mathrm{H}$ NMR measurements were attempted to define the reactive sites on PMPrS and to clarify the structure of PMPrS- $g$-PMMA, but resulted in little success. The ESR result suggests that alkyl radicals were generated at room temperature, but it is difficult to determine exact positions of those radicals. In ${ }^{1} \mathrm{H}$ NMR measurement, it is also hard to specify the position where graft chains attach to the main chain.

Therefore, we assumed the structure of radiation-modified polysilane by reference to other studies on radiation chemistry of polysilane. Seki et al. (1996) reported the $\gamma$-ray-induced process on poly(dimethylsilane) by ESR study. They demonstrated that radicals are generated on silicon atoms by elimination of side-chain methyl radicals 
upon $\gamma$-irradiation at $77 \mathrm{~K}$. Kumagai et al. (1996) studied the effect of $\gamma$-irradiation on poly(diethylsilane) and poly(cyclohexylmethylsilane) at $77 \mathrm{~K}$. They clarified that radicals are generated on alkyl side chains of these polysilanes by homolytic cleavage of $\mathrm{C}-\mathrm{H}$ bonds. In both cases, the initial process caused by $\gamma$-irradiation is the formation of radicals at an alkyl side-chain position. In consideration of the similar structure having relatively longer alkyl side chains, Kumagai’s case is more safely applicable to our case. Although the radicals often cause the homolytic cleavage of Si-Si bonds at elevated temperatures and therefore block type copolymers might be formed, main products are considered to be copolymers with grafted chains growing from alkyl side chains of PMPrS since radicals initially formed at a side-chain position can facilely attack surrounding MMA monomers.

For quantitative discussion, two indexes were evaluated: the degree of polymerization of PMMA graft chains, $d_{\mathrm{PMMA}}$, and the number of PMMA graft chains per silicon atom, $n_{\text {PMMA. }}$ It is possible to evaluate $d_{\text {PMMA }}$ from the ratio of the number of MMA units to that of PMMA chain ends and $n_{\mathrm{PMMA}}$ from the ratio of the number of PMMA chain ends to methyl-n-propylsilane (MPrS) units. Two signals observed at $\delta 5.5$ and $6.2 \mathrm{ppm}$ in ${ }^{1} \mathrm{H}$ NMR spectra of PMPrS- $g$-PMMA reveal that the terminal unit of PMMA graft chains has an unsaturated bond due to termination by disproportionation. Contrary to 
our expectations, a chain transfer reaction from growing PMMA chains to solvent toluene molecules during irradiation is negligible, judging from the absence of toluene-derived signals in ${ }^{1} \mathrm{H}$ NMR spectra. Other terminal structures of PMMA graft chains should exist, but signals due to them could not be clearly distinguished in ${ }^{1} \mathrm{H}$ NMR spectra because of interference by other signals. In this study, consequently, PMMA terminal units were counted by measuring the signals from the unsaturated bond.

For the following analysis based on ${ }^{1} \mathrm{H}$ NMR spectra, the ratio of integral intensity for a methoxy proton of PMMA ( $\delta 3.7 \mathrm{ppm})$ to that for a methylene proton of the unsaturated terminal of PMMA ( $\delta 6.2 \mathrm{ppm})$ is expressed as $r_{\mathrm{d} 1}$, and the ratio of integral intensity for a methylene proton of the unsaturated terminal of PMMA to that for a methyl proton of PMPrS ( $\delta 0.25 \mathrm{ppm})$ as $r_{\mathrm{n} 1}$. In addition, the number of the unsaturated terminals per graft chain is symbolized by $N_{\text {PMMA. }}$ Because the absolute value of $N_{\text {PMMA }}$ is hard to be determined by ${ }^{1} \mathrm{H}$ NMR measurement, we assumed $N_{\text {PMMA }}$ as 1.0 for each PMPrS- $g$-PMMA. Then, the degree of polymerization of PMMA chains $\left(d_{\text {PMMA }}\right)$ and the number of PMMA chains per silicon atom ( $\left.n_{\text {PMMA }}\right)$ are described as $d_{\mathrm{PMMA}}=r_{\mathrm{d} 1} N_{\mathrm{PMMA}}=r_{\mathrm{d} 1}$ and $n_{\mathrm{PMMA}}=r_{\mathrm{n} 1} / N_{\mathrm{PMMA}}=r_{\mathrm{n} 1}$, respectively. Figure 4 depicts the dependence of $d_{\mathrm{PMMA}}$ and $n_{\mathrm{PMMA}}$ on the concentration of MMA in the graft 
polymerization. As shown in the figure, $d_{\text {PMMA }}$ rises with increasing concentration of MMA, but $n_{\text {PMMA }}$ scarcely changes. These results demonstrate that the rise of grafting yield with an increase of MMA concentration (Figure 3) is due to the growth of the degree of polymerization but not to the growth of the number of graft chains.

Figure 4. Dependence of $d_{\mathrm{PMMA}}$ and $n_{\mathrm{PMMA}}$ on the concentration of MMA. The concentration of PMPrS was $10 \mathrm{wt} \%$ and dose rate was $1.9 \mathrm{kGy} / \mathrm{h}$. Closed and open symbols indicate $d_{\text {PMMA }}$ and $n_{\text {PMMA }}$, respectively. Circles and squares corresponds to doses of 40 and $95 \mathrm{kGy}$, respectively.

Figure 5 shows the dependence of $d_{\mathrm{PMMA}}$ and $n_{\mathrm{PMMA}}$ on dose. The $d_{\mathrm{PMMA}}$ decreases with increasing dose, which indicates that PMMA graft chains are degraded more readily at higher doses. In contrast, $n_{\text {PMMA }}$ rises with an increase of dose, showing that PMMA graft chains increase in number. These results reveal that the rise of grafting yield with increasing dose, which is shown in Figure 2a, is mainly caused by the increase of a graft density.

Figure 5. Dependence of $d_{\mathrm{PMMA}}$ and $n_{\mathrm{PMMA}}$ on dose. The concentrations of PMPrS and MMA were both $10 \mathrm{wt} \%$. Closed and open symbols indicate $d_{\mathrm{PMMA}}$ and $n_{\mathrm{PMMA}}$, 
respectively. Circles and squares correspond to dose rates 3.1 and $1.9 \mathrm{kGy} / \mathrm{h}$, respectively.

If $M_{\mathrm{n}}$ of PMPrS1 in Table 1 is used, the number of PMMA chains per PMPrS chain is estimated as ca. 0.32 for PMPrS-g-PMMA with grafting yield of 0.33 . The cause of such a low graft density is explained as follows. In PMMA graft polymerization, the initiation reaction by $\gamma$-rays is considerably slower than the chain propagation reaction. Then, PMMA chains grown at an early stage of the reaction sterically cover other initiation sites on the same polysilane chain. Due to this steric hindrance by graft chains, monomers can hardly access to the reactive sites and the grafting from these sites scarcely occurs. As a result, the graft density becomes low.

Therefore, it is required to depress the steric hindrance by use of a monomer with a small propagation rate constant to attain high graft density.

\subsection{Grafting Yield of PMPrS-g-PDEF}

DEF was employed as a grafting monomer with smaller propagation rate constant $\left(k_{\mathrm{p}}\right.$ $\left.=0.21 \mathrm{Lmol}^{-1} \mathrm{~s}^{-1}\right)$ than that of MMA $\left(k_{\mathrm{p}}=290 \mathrm{Lmol}^{-1} \mathrm{~s}^{-1}\right)$ (Leonard, 1999; Otsu et al., 1991). The dependence of grafting yield of PMPrS-g-PDEF on dose and dose rate is 
shown in Figure 6. Grafting yield rises with an increase of dose in Figure 6a as in the case of PMPrS-g-PMMA, but the leveling-off behavior at higher doses as observed for PMPrS-g-PMMA is hardly recognized. This result can be explained as follows. Reduction of DEF monomers is more moderate than that of MMA monomers because DEF has the smaller propagation rate constant and the amount of DEF consumed by homopolymerization is small. As a result, more monomers are available for graft polymerization compared to the case of PMPrS-g-PMMA.

On the other hand, grafting yield of PMPrS-g-PDEF decreases with increasing dose rate in Figure 6b. This relationship between grafting yield and dose rate is consistent with that shown in Figure 2b. DEF has a smaller propagation rate constant than MMA does, but the competition between graft polymerization and homopolymerization also occurs as in the case of PMPrS- $g$-PMMA.

Figure 6. Dependence of grafting yield of PMPrS-g-PDEF on (a) dose and (b) dose rate. The concentrations of PMPrS and DEF were 10 and $30 \mathrm{wt} \%$, respectively.

Figure 7 depicts the dependence of grafting yield on the concentration of DEF. Similarly to the case of PMPrS-g-PMMA, grafting yield rises with increasing 
concentration of DEF.

Figure 7. Dependence of grafting yield of PMPrS- $g$-PDEF on the concentration of DEF. The concentration of PMPrS was $10 \mathrm{wt} \%$. Dose rate was $2.0 \mathrm{kGy} / \mathrm{h}$ and dose was $40 \mathrm{kGy}$.

\subsection{Characterization of PMPrS-g-PDEF}

In ${ }^{1} \mathrm{H}$ NMR spectra of PMPrS-g-PDEF, a signal owing to PDEF chain ends was observed at $\delta$ 6.7-6.9 ppm. Other signals due to the chain ends were not distinguished. The observed signals indicate that the terminal unit of PDEF graft chains has an unsaturated structure.

In the same manner described above, we hereafter use the following quantities: the degree of polymerization of PDEF graft chains, $d_{\mathrm{PDEF}}$, the number of PDEF graft chains per silicon atom, $n_{\mathrm{PDEF}}$, and the number of the unsaturated terminals, $N_{\text {PDEF. }}$ The following values are also defined: $r_{\mathrm{d} 2}$ as the ratio of integral intensity for a methylene proton of PDEF ( $\delta 4.2 \mathrm{ppm})$ to that for a methine proton of the unsaturated terminals $(\delta$ 6.7-6.9 $\mathrm{ppm}$ ), and $r_{\mathrm{n} 2}$ is one for a methine proton of the unsaturated terminals to that of a methyl proton of PMPrS. As in the case of PMPrS- $g$-PMMA, we assume $N_{\text {PDEF }}$ as 1.0 for each PMPrS- $g$-PDEF. Then, $d_{\mathrm{PDEF}}$ and $n_{\mathrm{PDEF}}$ are expressed by $d_{\mathrm{PDEF}}=r_{\mathrm{d} 2}$ and 
$n_{\mathrm{PDEF}}=r_{\mathrm{n} 2}$, respectively.

Figure 8 shows the dependence of $d_{\mathrm{PDEF}}$ and $n_{\mathrm{PDEF}}$ on dose. Contrary to the $d_{\mathrm{PMMA}}$ of PMPrS- $g$-PMMA, $d_{\text {PDEF }}$ rises with increasing dose. This indicates that the PDEF chains are less degradable than PMMA chains and the growth of PDEF chains is predominant over the degradation except at higher doses. In Figure 9, $n_{\text {PDEF }}$ also rises with increasing dose. The rise of grafting yield of PMPrS- $g$-PDEF shown in Figure 6a is due to the increase of both the degree of polymerization and the number of grafted chains, unlike the PMPrS- $g$-PMMA case.

Figure 8. Dependence of $d_{\mathrm{PDEF}}$ (closed circles) and $n_{\mathrm{PDEF}}$ (open circles) on dose. The concentrations of PMPrS and DEF were 10 and $30 \mathrm{wt} \%$, respectively. Dose rate was $2.0 \mathrm{kGy} / \mathrm{h}$.

For further quantitative discussion, we estimated the graft density of PMPrS-g-PDEF in the same way as shown above.

If $M_{\mathrm{n}}$ of PMPrS2 in Table 1 is employed, the number of PDEF chains per PMPrS chain is estimated as ca. 0.62 for PMPrS-g-PDEF with grafting yield of 0.34 . Although the graft density of PMPrS- $g$-PDEF is higher than that of PMPrS- $g$-PMMA, this value is still lower than we have expected. The propagation of PDEF graft chains 
is still too fast to overcome steric hindrance by grafted chains. Therefore, we adopted a monomer with no ability of homopolymerization, MAH.

\subsection{Grafting Yield of PMPrS-g-MAH}

Because MAH is a non-homopolymerizable monomer in radical polymerization (Hodge et al., 1985, 1990), it is expected that the steric hindrance by grafted chains is reduced and the higher graft density of PMPrS-g-MAH is attained compared with that of PMPrS- $g$-PMMA and PMPrS- $g$-PDEF.

Prior to using MAH for graft polymerization, we confirmed by GPC and ${ }^{1} \mathrm{H}$ NMR measurement that MAH does not homopolymerize indeed by $\gamma$-irradiation.

Figure 9 depicts the dependence of grafting yield of PMPrS-g-MAH on dose. The grafting yield of PMPrS-g-MAH rises with increasing dose. The rise of grafting yield directly indicates the rise of graft density of PMPrS- $g$-MAH because the grafted chains have only one MAH unit.

The number of grafted MAH per silicon atom, $n_{\mathrm{MAH}}$, is calculated by multiplying grafting yield by the number of MPrS units of PMPrS. For PMPrS- $g$-MAH with grafting yield of $0.24, n_{\mathrm{MAH}}$ is calculated as 4.2 ; in other expression, the number of MAH bonded to one PMPrS chain is $c a$. 30. It is thus demonstrated that higher graft 
density is attained by MAH grafting as we expected.

Figure 9. Dependence of grafting yield of PMPrS-g-MAH on dose. The concentrations of PMPrS and MAH were $10 \mathrm{wt} \%$. Dose rate was $2.0 \mathrm{kGy} / \mathrm{h}$.

With an aim to attain larger grafting yield, i.e., higher graft density, higher dose of $\gamma$-rays was irradiated for synthesizing PMPrS-g-MAH. The results are summarized in Table 2. Grafting yield listed in Table 2 is higher than that shown in Figure 9. However, UV absorption spectra of these PMPrS-g-MAH samples show peak shifts of $\sigma-\sigma^{*}$ absorption to shorter wavelength, which reflects the degradation of PMPrS main chains by high dose of $\gamma$-rays.

\section{Table 2.}

We used higher concentration of MAH to attain higher grafting yields, but PMPrS could not be dissolved in a toluene solution of MAH with a concentration above $11 \mathrm{wt} \%$. Accordingly, relative concentration of MAH with reference to PMPrS was increased by reducing the concentration of PMPrS for a fixed MAH concentration. However, grafting yield was almost constant even with changing the concentration of PMPrS. 
This result presumably indicates that the rate-determing step is generation of reactive sites on PMPrS and the grafting rate of MAH is independent of the concentration of PMPrS. As a result, the grafting yield is also irrelevant to the concentration of PMPrS. In the case of PMPrS- $g$-PMMA and PMPrS- $g$-PDEF, on the other hand, grafting yield depends on the concentration of grafting monomers as discussed above. Generation of reactive sites on PMPrS would be a rate-determining step also in this case, but the grafting yield of these polymers is determined by graft chain length which is subject to monomer concentration.

\section{Conclusion}

We demonstrated that $\gamma$-ray-induced graft polymerization is the simple but effective method for the modification of polysilane. For the grafting of MMA and DEF to PMPrS, grafting yield rises with increasing dose and concentrations of the monomers, but the number of grafted chains per PMPrS chain was estimated to be less than 1.0. Such low graft density was due to the steric hindrance of growing grafted chains on PMPrS. To depress the steric hindrance and increase the graft density of modified PMPrS, non-homopolymerize monomer MAH was used not to form bulky grafted chains. With an increase of dose, grafting yield of PMPrS-g-MAH and consequently 
the graft density increases as we have expected. The highest graft density obtained in this study was one MAH unit for 4.2 silicone atoms of a PMPrS backbone.

In this way, we revealed the structure of radiation-modified polysilane-based graft copolymers. In particular, the graft chain length and density were clarified. It is also demonstrated that grafting yield of radiation-modified polysilane can be controlled by the irradiation conditions such as dose, dose rate and concentration of monomers.

\section{References}

Alexandridis, P., Lindman, B., 2000. Amphiphilic Block Copolymers: Self-Assembly and Application, Elsevier, Amsterdam.

Hodge, P., Khoshdel, E., Tredgold, R. H., Vickers, A. J., Winter, C. S., 1985. Langmuir-blodgett films from preformed polymers British Polymer Journal 17, 368-371.

Hodge, P., Davis, F., Tredgold, R. H., Silver, J., Coles, H., 1990. Preformed Polymers for Langmuir-Blodgett Films. J. Phil. Trans. R. Soc. Lond. A330, 153-166. 
Kumagai, J., Oyama, K., Yoshida, H., Ichikawa, T., 1996. Effect of ionizing radiation on polysilane. Radiat. Phys. Chem. 47, 631-636.

Leonard, J., 1999. Polymer Handbook 4th Edition; Brandrup, J., Immergut, E. H., Grulke, E. A. (Eds.), Wiley, New York, p. 87.

Miller, R. D., Michl, J., 1989. Polysilane high polymers. Chem. Rev. 89, 1359-1410.

Otsu, T., Ymada, B., Ishikawa, T., 1991. Determination of absolute rate constants for elementary reactions in radical polymerization of diethyl fumarate. Macromolecules 24, 415-419.

Riess, G., 2003. Micellization of block copolymers. Prog. Polym. Sci. 28, 1107-1170.

Seki, S., Tagawa, S., Ishigure, K., Cromack, K. R., Trifunac, A. D., 1996. Observation of silyl radical in $\gamma$-radiolysis of solid poly(dimethylsilane). Radiat. Phys. Chem. 47, 217-219. 
Webber, S. E., Munk, P., Tuzar, Z., 1996. Solvents and Self-Organization of Polymers, Kluwer, The Netherlands.

West, R. J., 1986. The polysilane high polymers. J. Organomet. Chem. 300, 327-346.

West, R., 1992. Polysilanes and Related Polymers, in: Mark, J. E., Allcock, H. R., West, R. (Eds.), Inorganic Polymers. R. Prentice Hall, Englewood Cliffs, NJ. 
Table 1. Number average molecular weight $\left(M_{\mathrm{n}}\right)$ and polydispersity index (PDI) of PMPrS.

\begin{tabular}{ccc}
\hline & $M_{\mathrm{n}} \times 10^{-4 \mathrm{a}}$ & $\mathrm{PDI}^{\mathrm{b}}$ \\
\hline \hline PMPrS1 & 1.3 & 2.4 \\
PMPrS2 & 1.2 & 3.8 \\
PMPrS3 & 1.1 & 3.8 \\
\hline
\end{tabular}

$\overline{\mathrm{a}, \mathrm{b}}$ Determined by GPC (RI detector) with polystyrene standards and a THF eluent. 
Table 2. Grafting yield of PMPrS-g-MAH at higher doses. The concentrations of PMPrS and MAH were both $10 \mathrm{wt} \%$.

\begin{tabular}{rcc}
\hline $\begin{array}{r}\text { Dose } \\
(\mathrm{kGy})\end{array}$ & $\begin{array}{c}\text { Dose rate } \\
(\mathrm{kG} / \mathrm{h})\end{array}$ & Grafting yield \\
\hline 275 & 5.0 & 0.32 \\
550 & 10.0 & 0.50 \\
1100 & 20.0 & 0.62 \\
\hline
\end{tabular}




\section{A Self-archived copy in 京都大学学術情報リポジトリ}

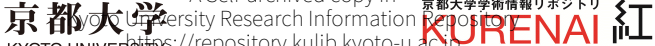

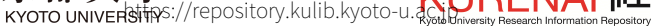

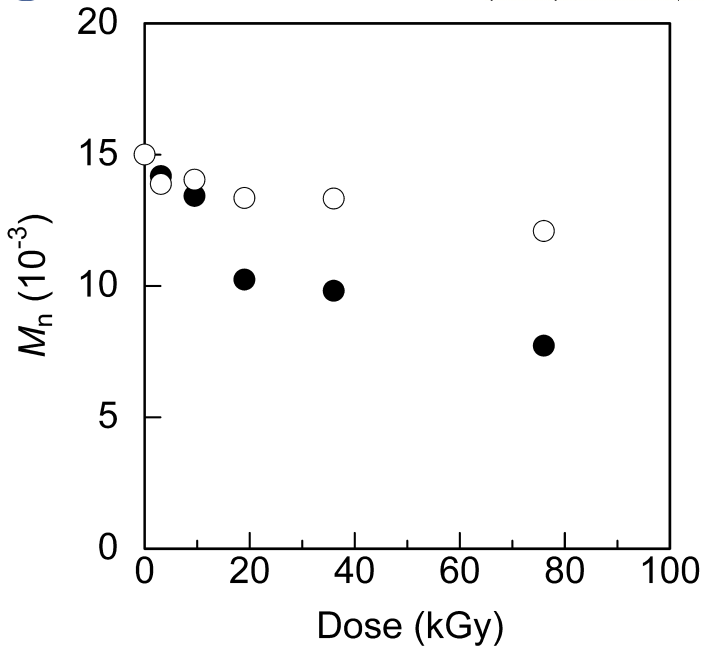



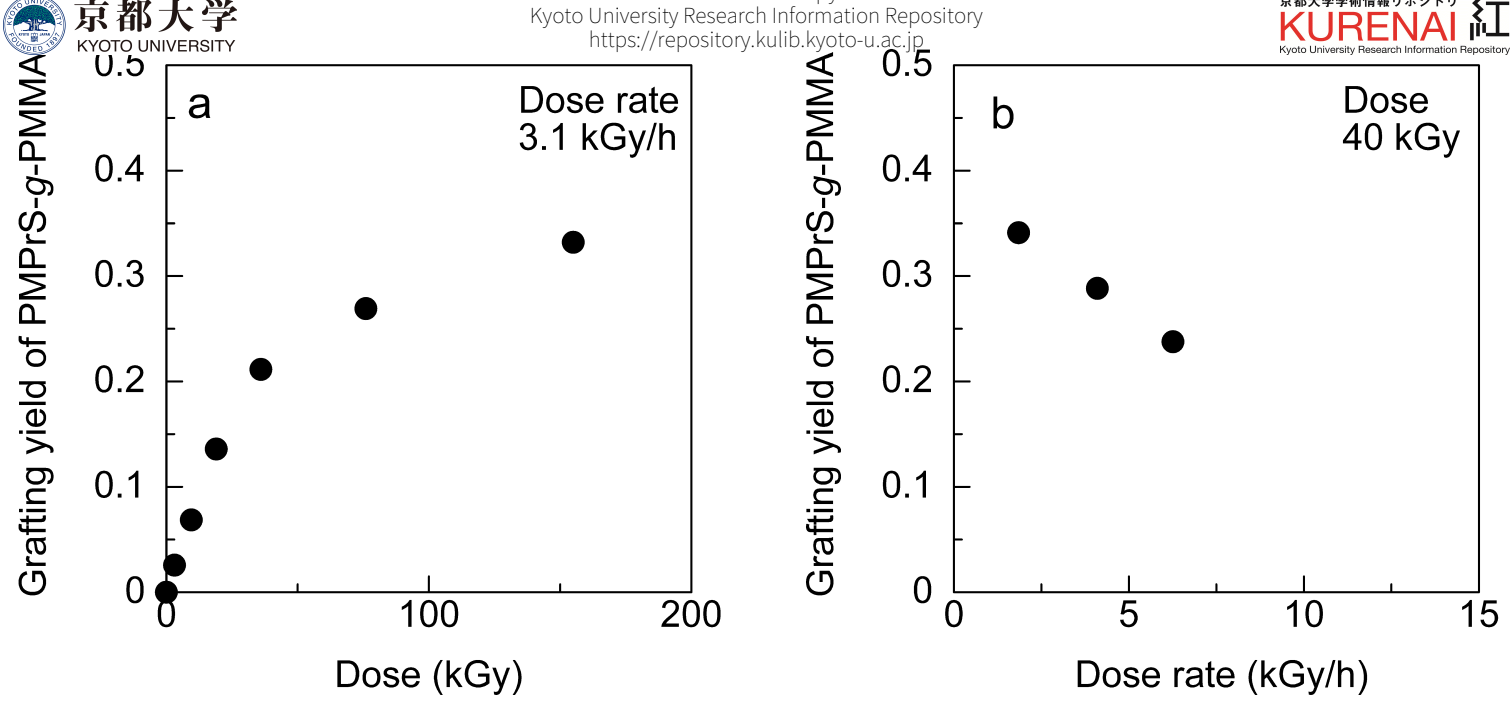
A Self-archived copy in 京都大学学術情報リポジトリ 宗 都

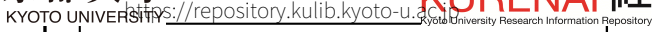

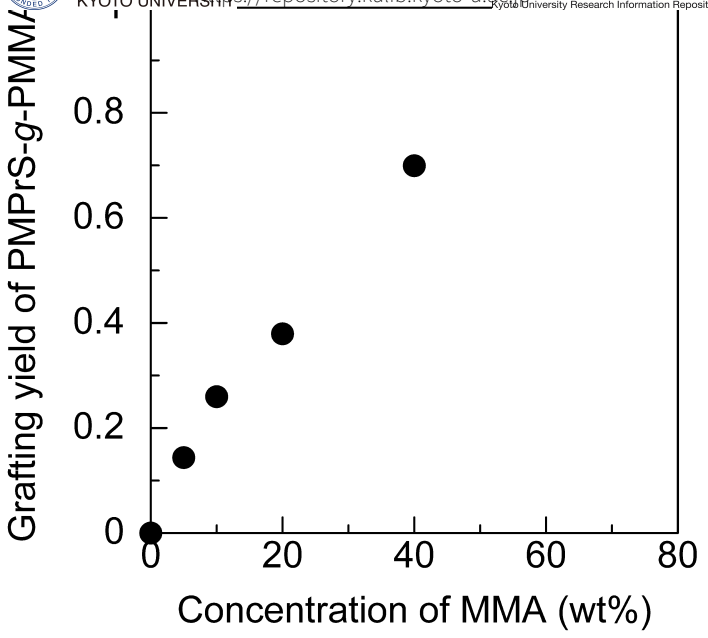




\section{A Self-archived copy in 京都大学学術情報リポジトリ}

S

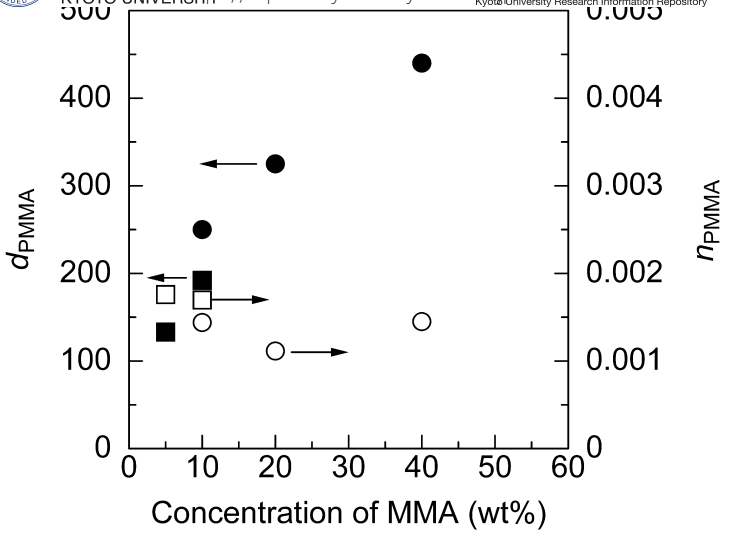




\section{A Self-archived copy in 京都大学学術情報リポジトリ}

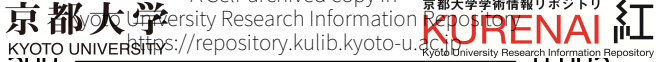

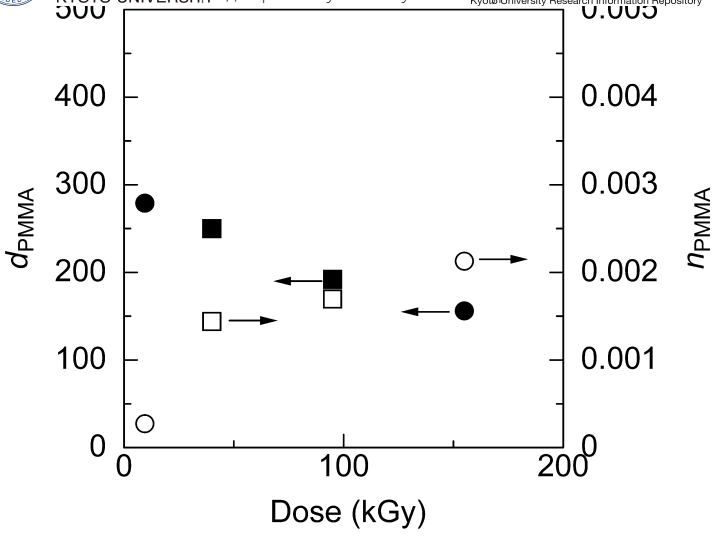


票都大学

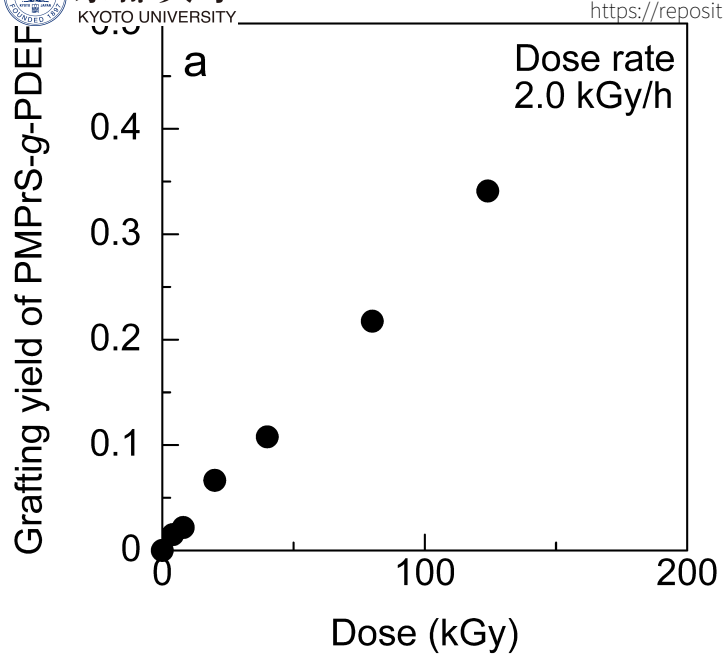

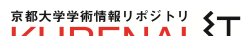
KURENAI iII

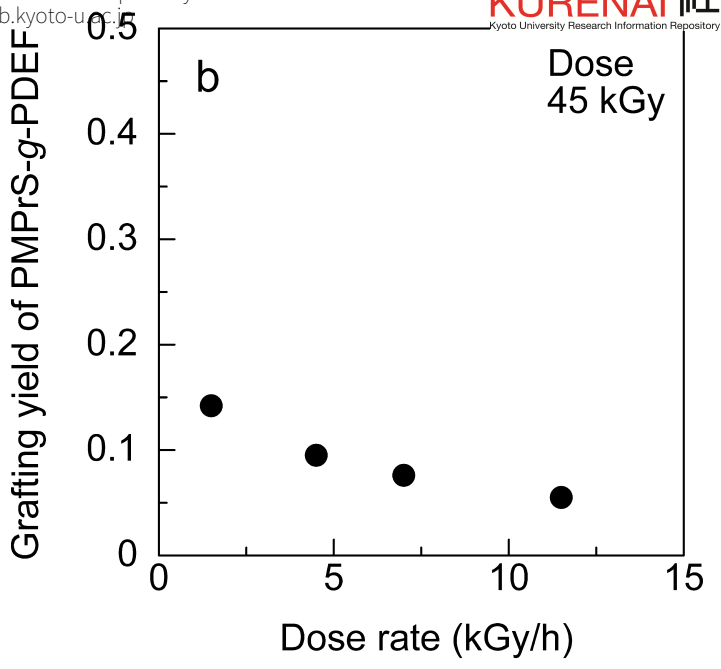




\section{A Self-archived copy in 京都大学学術情報リポジトリ}

宗 都

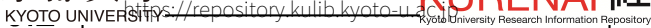

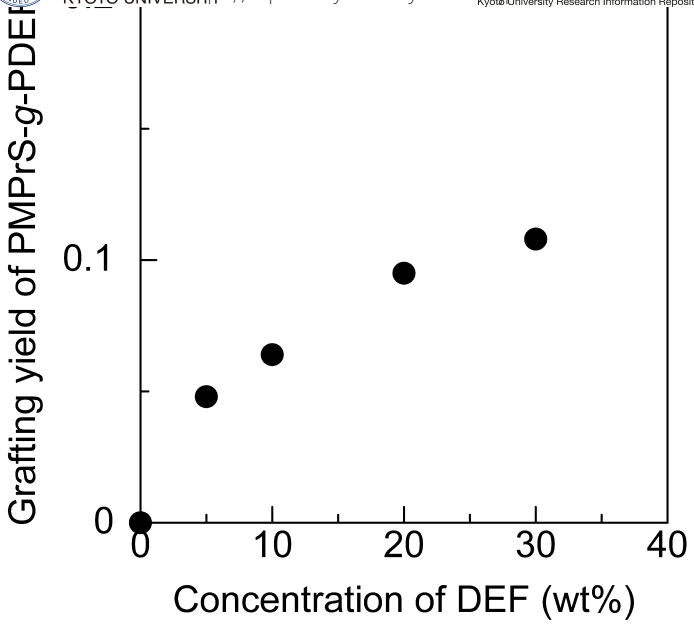


A Self-archived copy in 京都大学学術情報リポジトリ SY

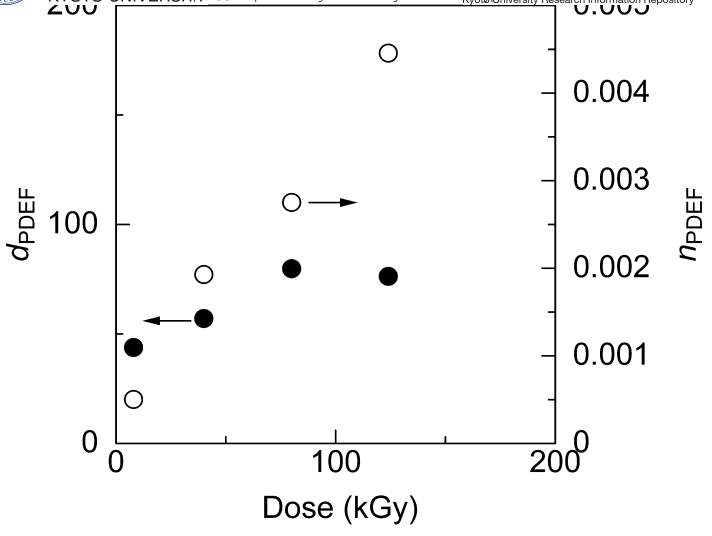


A Self-archived copy in 京都大学学術情報リポジトリ SY

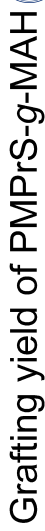
U. 